\title{
Konventionelle Systemtherapie statt Biologika?
}

Biologika sind in der Therapie der mittelschweren und schweren Psoriasis etabliert. Doch nach wie vor ist die konventionelle Systemtherapie die Erstlinienoption. Prof. Dr. Jörg Prinz, Klinik und Poliklinik für Dermatologie und Allergologie, LMU München, stellte sie im Rahmen der FOBI 2020 auf den Prüfstand.

„Die konventionelle Systemtherapie ist nicht ganz so verlässlich wie Biologika, aber oft eine gute therapeutische Möglichkeit", so Prinz mit Blick auf die aktuellen Leitlinienempfehlungen und die Wirtschaftlichkeit.

\section{MTX: auf renale Elimination achten} Methotrexat (MTX) ist aus Sicht von Prinz eine sichere Langzeittherapie der Psoriasis und der Psoriasisarthritis. Zwingend beachtet werden muss allerdings die renale Elimination, um eine Myelosuppression zu verhindern. Denn eine verzögerte renale Ausscheidung führt zu einer intrazelIulären MTX-Akkumulation und damit zu einer relativen Überdosierung. Neben Nierenfunktionsstörungen können auch Arzneimittelwechselwirkungen zu einer vermehrten intrazellulären MTX-Aufnahme führen. Zu den relevanten Arzneimitteln mit Gefahr einer relativen MTX-Überdosierung gehören unter anderem nicht-steroidale Antirheumatika. Klinische Alarmzeichen einer beginnenden Agranulozytose und Thrombopenie sind unter anderem die nekrotisierende Angina und Gingivitis sowie Schleimhautund Hauteinblutungen. Dann, so Prinz, kann es zu einem raschen Übergang in eine lebensbedrohliche Sepsis kommen.

\section{Acitretin im gebärfähigen Alter verboten}

Acitretin ist laut Prinz vor allem zur Kombinationstherapie der chronischen Plaque-Psoriasis mit Phototherapie oder Biologika geeignet und gilt als Therapie der Wahl für papulopustulöse Psoriasisformen (• Abb. 1). Als Retinoid ist es wegen seiner Teratoge- nität bei Frauen im gebärfähigen Alter kontraindiziert.

\section{\) Bei MTX muss die renale}

Elimination beachtet werden, um eine Myelosuppression zu verhindern

Fumarsäure: individuelle Dosierung Als Herausforderung beim Einsatz von Fumarsäurestern nannte Prinz die individuelle Dosisfindung. Es gebe keine erkennbare Beziehung zwischen Dosis, Wirkung und Nebenwirkungen. Die wirksame und verträgliche Dosis müsse für jeden Patienten individuell etabliert werden. „Dabei müssen es nicht sechs Tabletten am Tag sein", so Prinz. Gastrointestinale Beschwerden wie Bauchschmerzen und Diarrhoe, aber auch Flushing können eine Dosisreduktion erforderlich machen. Wichtig ist auch der Blick auf das Blutbild. Eine Eosinophilie ist in der Regel unbedenklich. Bei einem Anteil von über $20 \%$ an den Leukozyten sollte aber die Dosis reduziert, gegebenenfalls die Therapie beendet werden. Auch das Risiko einer Lymphopenie, die meist innerhalb der ersten drei Monate nach Therapiebeginn, spätestens nach etwa zwölf Wochen auftritt, muss im Auge behalten werden.

\section{Ciclosporin nach Idealgewicht dosieren}

Ciclosporin kann bei schwerer Psoriasis eingesetzt werden bei Patienten, bei denen eine herkömmliche Therapie nicht geeignet oder nicht wirksam ist. $\mathrm{Zu}$ beachten ist auch hier die richtige Dosierung. Empfohlen wird eine Dosis von $2,5 \mathrm{mg}$ bis $5 \mathrm{mg}$ pro kg Körpergewicht, allerdings bezogen auf das Idealgewicht entsprechend der Kör- pergröße, nicht auf das Realgewicht. Prinz empfahl eine Startdosis von 3,5 bis $4 \mathrm{mg} / \mathrm{kg}$ Idealgewicht, um ein rascheres Ansprechen zu erreichen, mit anschließender Dosisreduktion. Erreichen lässt sich, unter Beachtung auch von Medikamenteninteraktionen, eine sichere Remissonsinduktion. "Es ist aber nicht geeignet für die Langzeittherapie."

Hinweis des Verlags. Der Verlag bleibt in Hinblick auf geografische Zuordnungen und Gebietsbezeichnungen in veröffentlichten Karten und Institutsadressen neutral.

hautnah $2020 \cdot 19: 89$

https://doi.org/10.1007/s12326-02000394-7

๑) Springer-Verlag GmbH Austria, ein Teil von Springer Nature 2020

Quelle: Dr. Beate Fessler, SpringerMedizin.de (basierend auf: Praktisches Krankheitsmanagement: Systemtherapie der Psoriasis vulgaris, 27. Fortbildungswoche für praktische Dermatologie und Venerologie (FOBI Digital), 7. bis 11.07.2020)

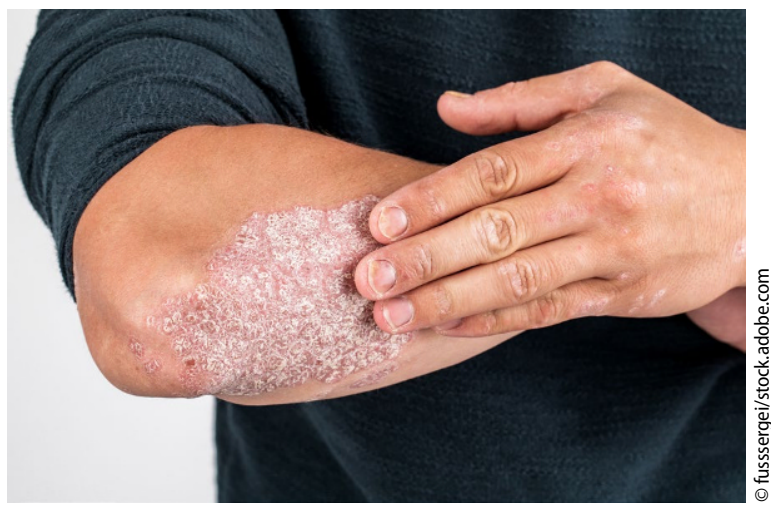

Abb. $1 \Delta$ Acitretin eignet sich vor allem zu einer Kombinationstherapie der chronischen Plaque-Psoriasis. (Symbolbild mit Fotomodell) 